\title{
Preparation and characterization of some unsymmetrical 2-(dialkylamino)ethanethiols
}

\author{
Jakub Stýskala, ${ }^{a}$ Petr Cankař, ${ }^{\text {a* }}$ Miroslav Soural, ${ }^{a}$ Petr Bednář, ${ }^{b}$ and Karel Lemr ${ }^{b}$ \\ ${ }^{a}$ Department of Organic Chemistry, Palacký University, Tř. Svobody 8, 77146 Olomouc, \\ Czech Republic \\ ${ }^{b}$ Department of Analytical Chemistry, Palacký University, Tř. Svobody 8, 77146 Olomouc, \\ Czech Republic \\ E-mail: cankar@orgchem.upol.cz
}

\begin{abstract}
The synthesis of some 2-(dialkylamino)ethanethiols is described. A method involving formation of isothiouronium salts from 2-(dialkylamino)ethyl chlorides by the reaction with thiourea followed by the alkaline hydrolysis has been chosen. Prepared compounds can be used as the standards for the analytical chemical methods to monitor the chemical warfare agents and their precursors or metabolites.
\end{abstract}

Keywords: 2-(Dialkylamino)ethyl chloride, 2-(dialkylamino)ethanethiol, precursors of chemical warfare agents

\section{Introduction}

The monitoring of the production, movement and destruction of chemical warfare agents (CWAs) and their precursors is an important task nowadays regarding the ubiquitous terroristic and military activities. The related analytical control involves first of all the methods providing fast and reliable information about presence and concentration of a hazardous compound itself to allow a "real time" sufficient response in politically or militarily sensitive situations. Beside the methods for unambiguous control of precursors and side products of synthesis or degradation are important tools to block the prohibited movement of the base material for the CWA synthesis and to control the directed disposal. Another essential task is to prove the using of the CWA in the target locality or the exposition of a human subject (e.g. identification of products of their

transformation) ${ }^{1}$. The majority of these activities and tasks are defined and listed in The Convention on the Prohibition of the Development, Production, Stockpiling and Use of Chemical Weapons and on their Destruction (OPCW) ${ }^{2}$. 
2-(Dialkylamino)ethanethiols (Me, Et, n-Pr, iPr) are listed in the Schedule 2 B Precursors of the Convention. The Schedule comprises precursors in one of the chemical reactions at the final stage of CWA formation or compounds with the CWA capacity ${ }^{2}$. These thiols are formed also during hydrolysis of highly toxic nerve agents known as $\mathrm{V}$-agents as described in the Scheme $1^{3-5}$. Two V-agents were extensively produced; i.) VX (S-2-(diisopropylamino)ethyl Oethyl methylphosphonothioate in United States and ii.) R-VX (Russian VX, S-2(diethylamino)ethyl O-ethyl methylphosphonothioate) in Russia ${ }^{3}$. Obviously the N-substitution can influence the toxicity of $\mathrm{V}$-agent (i.e. transport through biomembranes and effectiveness of the reaction with acetylcholinesterase as common target in body cells) as well as the hydrolysis rate in the environment.

Considering the relatively low cost and low technology to produce these chemical weapons ${ }^{5}$ and consequently potential risk of appearence of all possible derivatives (different substitutions) a comprehensive study of all exploitable thiols is of a high importance. However, the development of related analytical methods allowing such a research (first of all high performance separation methods and their combination with mass spectrometry) is dependent on the availability of high quality standards.
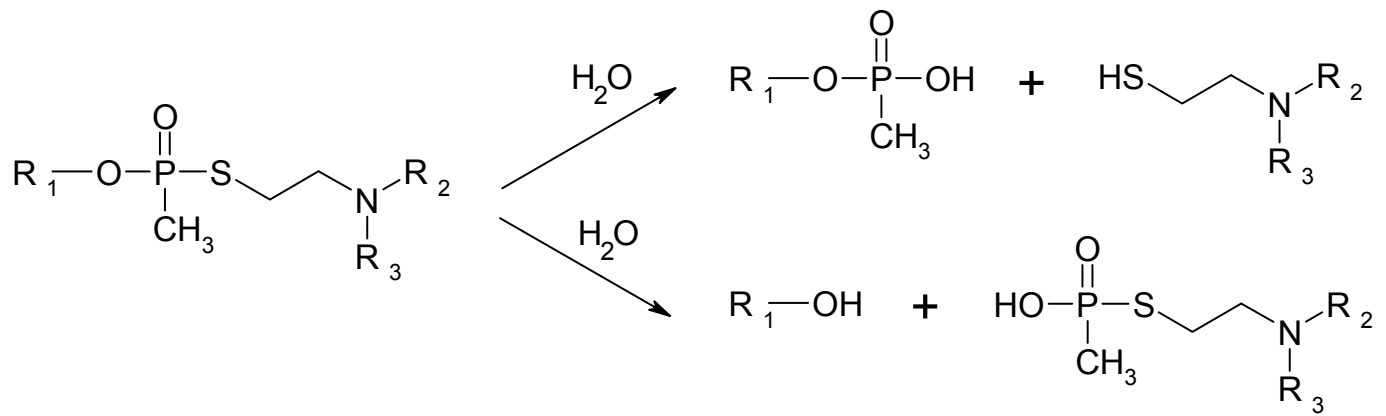

Scheme 1. Process of V-agents hydrolysis.

2-(Dialkylamino)ethanethiols of general formula $\mathrm{R}_{1} \mathrm{R}_{2} \mathrm{NCH}_{2} \mathrm{CH}_{2} \mathrm{SH}$ are possible to prepare by the three general methods. The first one involves the reaction of 2-(dialkylamino)ethyl halides with the hydrosulfides of the alkali metals ${ }^{6,7}$. Another method is based on the nucleophilic substitution of the 2-(dialkylamino)ethyl halides with thiourea followed by an alkaline hydrolysis of the isothiouronium salts ${ }^{8,9}$ and the last method is the mercaptoethylation of the primary and the secondary amines with ethylene sulfide ${ }^{10-12}$ or other mercaptoethylating agents such as ethyl 2-hydroxyethylthiolcarbonate 13-15, ethyl 2-mercaptoethylcarbonate ${ }^{13-15}$ and ethylene monothiolcarbonate ${ }^{16}$.

Although some derivatives of 2-(dialkylamino)ethanethiols are cited in the literature, the references mostly describe them as a starting material for the next reaction step without sufficient or even any characterization or description of the preparation. Hence, this communication is not only devoted to the synthesis of the novel 2-(dialkylamino)ethanethiols, 
but also to the derivatives that have been already cited without sufficient description of the preparative method or characterization. Moreover, in the case of derivatives where the procedure has been already described we have offered an alternative methodology of the preparation. The 2-(dialkylamino)ethanethiols will be used as standards for analytical methods, therefore, a higher priority has been given to the chemical purity of the prepared derivatives than to reach the highlevel yields in the particular reaction steps of the procedure.

\section{Results and Discussion}

Regarding the difficulties with the synthesis of unsymmetrical secondary amines as a starting material for the mercaptoethylation with ethylene sulfide or similar agents we have applied the synthetic method using the hydrolysis of isothiouronium salts (Scheme 2.) which can afford satisfactory yields even on the microscale. This procedure is better than methods using hydrosulfide.

Most of the 2-(dialkylamino)ethyl chlorides have been already described ${ }^{17,18}$, only for the derivatives $\mathbf{2 b}$ and $\mathbf{2 d}$ has there not been found any reference. These chlorides have been prepared by the treatment of the corresponding alcohols $\mathbf{1} \mathbf{b}^{22}$ and $\mathbf{1 d}{ }^{19}$ with thionyl chloride in chloroform. The proper cooling at this reaction step is necessary to avoid the formation of byproducts. Although the aminoalcohol $\mathbf{1 b}$ has been found in the literature we have decided to modify the preparation procedure. This compound was prepared by the alkylation of 2(methylamino)ethanol with 1-iodopropane in toluene. The alternative approach (the alkylation of 2-(propylamino)ethanol with iodomethane) has been also tested but the yields of the aminoalcohol 1b ranged only between 5-10\% because the quaternary ammonium salts were formed predominantly.

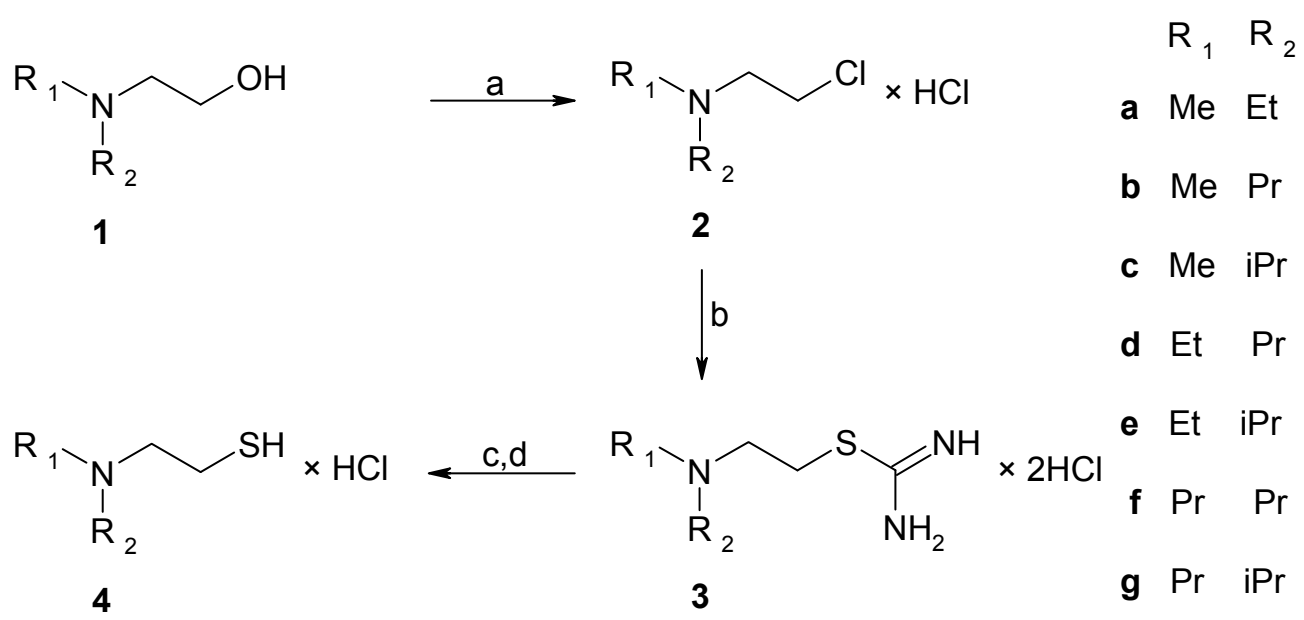

Scheme 2. (a) $\mathrm{SOCl}_{2}, \mathrm{CHCl}_{3}$; (b) thiourea, EtOH; (c) $\mathrm{NaOH}, \mathrm{H}_{2} \mathrm{O}$; d) $\mathrm{HCl} / \mathrm{EtOH}$. 
In the following step the isothiouronium group was introduced by the treatment of equimolar amount of the corresponding 2-(dialkylamino)ethyl chloride $\mathbf{2}$ and thiourea in the ethanolic solution. Since the similar isothiouronium salts are not fully characterized in the literature (only some references ${ }^{8,20,21}$ have been found for the symmetrically N-substituted derivatives which have not been isolated and characterized by the spectral methods but directly used for the next reaction step) we decided to isolate and characterize these compounds. Thus, dialkylaminoethylisothiouronium salts $\mathbf{3}$ have been isolated from the reaction mixture by the simple precipitation with diethyl ether and purified by the recrystallization from a mixture of dry ethanol and diethyl ether. The yields ranged between $72-90 \%$. The isothiouronium group was identified in the NMR spectrum by the presence of chemical shifts 9.49-9.56 ppm for the $\mathrm{NH}_{2}$ protons and 168.9-169.2 ppm for the carbon atom respectively. The dominant peak in the electrospray ionization mass spectra of the isothiuronium salts corresponded with the related diaminomethylene-[2-(dialkylamino)-ethyl]-sulfonium (quasimolecular ion, $[\mathrm{M}+\mathrm{H}]^{+}$). Beside, thermal fragments arising in the ion source (i.e. neutral loss of isothiourea, dialkylamines, elimination of $\mathrm{N}$-substituted alkyl chains and formation of related mercapto derivatives) were found in the spectra. Since the compounds were prepared as hydrochlorides, related adducts of two quasimolecular ions with chloride (i.e. $[2(\mathrm{M}+\mathrm{H})+\mathrm{Cl}]^{+}$) were detected in the spectra as well. The desired 2-(dialkylamino)ethanethiols 4 have been obtained by the alkaline hydrolysis of the corresponding isothiouronium salts 3 . This reaction had to be carried out in degassed solvents otherwise the formation of unwanted oxidative by-products of thiols occurred. The relatively low yields in this step (19-56\%) were largely due to the purification methods used which higher priority to purity of the sample than to a high-level yield. Distillation of the crude products was necessary and the best method (even though the derivatives with the lower mass can co-distill with diethyl ether ${ }^{8}$ ) for the separation of the free 2-(dialkylamino)ethanethiols 4 from the sulfide side-products which have been detected by the mass spectrometry after alkaline hydrolysis. All of the 2-(dialkylamino)ethanethiols 4 prepared have been converted to the corresponding hydrochlorides to provide better stability and handling with the samples.

Although the preparation of 2-(dipropylamino)ethanethiol $\mathbf{4 f}$ via the mercaptoethylation methods is known ${ }^{13-15}$, we decided to prepare this compound also by the alkaline hydrolysis of the corresponding isothiouronium salt $\mathbf{3 f}$ and characterized it by the standard spectroscopic methods. With respect to the yield the mercaptoethylation has been found as a better procedure than an alkaline cleavage of isothiouronium salt $\mathbf{3 f}$.

\section{Conclusions}

We have offered an alternative methodology for the preparation of unsymmetrically Nsubstituted 2-(dialkylamino)ethanethiols $\mathbf{4}$ using the alkaline hydrolysis of isothiouronium salts $\mathbf{3}$ which have been obtained from the corresponding 2-(dialkylamino)ethyl chlorides 2 . The prepared 2-(dialkylamino)ethanethiols 4 can be used as standards for analytical chemical 
methods to monitor the chemical warfare agents. Futhermore, the procedure could be extended to the synthesis of deuterated analogs which are useful in analytical chemical methods.

\section{Experimental Section}

General Procedures. Melting points were determined on a Boetius stage and are uncorrected. The IR spectra were recorded in $\mathrm{KBr}$ wafers on an ATI Unicam Genesis FTIR instrument. The NMR spectra were registered on a Bruker Avance $300 \mathrm{MHz}$ DRX spectrometer; chemical shifts are reported in ppm, the coupling constants $J$ in Hz. Elemental analyses were performed with an EA 1108 Elemental Analyser (Fison Instruments). Mass spectrometric experiments were performed using an LCQ ion trap mass spectrometer (Finnigan MAT, San Jose, CA, USA).

2-(Methylpropylamino)ethanol (1b). To a stirred solution of 2-(methylamino)ethanol (5.0 g, $66.6 \mathrm{mmol})$ in toluene $(10 \mathrm{ml})$ was added 1-iodopropane $(14.7 \mathrm{~g}, 86.6 \mathrm{mmol})$. After 10 minutes of stirring at room temperature the reaction mixture was heated to $60^{\circ} \mathrm{C}$ and stirred at this temperature for 16 hours. After cooling to a room temperature the mixture was diluted with water $(5 \mathrm{ml})$ and extracted with diethyl ether $(2 \times 3 \mathrm{ml})$ which was discarded. To remained water solution was added solution of $\mathrm{NaOH}(3.0 \mathrm{~g}, 75.0 \mathrm{mmol})$ in water $(4 \mathrm{ml})$. Then, the product was extracted with diethyl ether $(3 \times 10 \mathrm{ml})$ and dried with $\mathrm{MgSO}_{4}$. After evaporation of diethyl ether in vacuo, the crude oily product was purified by vacuum distillation. Yield $3.3 \mathrm{~g}(42 \%)$, MS (ESI, $\mathrm{m} / \mathrm{z}$ ): $118.1[\mathrm{M}+\mathrm{H}]^{+}$; IR $\left[\mathrm{cm}^{-1}\right]: 3412,2960,2874,2798,1468,1458,1042 .{ }^{1} \mathrm{H}-\mathrm{NMR}$ (DMSO- $d_{6}$ ): $\delta 0.84$ (t, 3H, $\mathrm{CH}_{3}, J=7.5 \mathrm{~Hz}$ ); 1.39 (sextet, $2 \mathrm{H}, \mathrm{CH}_{2}, J=7.5 \mathrm{~Hz}$ ); 2.14 (s, 3H, $\left.\mathrm{CH}_{3}\right) ; 2.26\left(\mathrm{t}, 2 \mathrm{H}, \mathrm{CH}_{2}, J=7.5 \mathrm{~Hz}\right) ; 2.37\left(\mathrm{t}, 2 \mathrm{H}, \mathrm{CH}_{2}, J=6.6 \mathrm{~Hz}\right) ; 3.45$ (t, 2H, $\mathrm{CH}_{2}, J=6.6$ $\mathrm{Hz}) ; 4.27$ (bs, $1 \mathrm{H}, \mathrm{OH}) ;{ }^{13} \mathrm{C}-\mathrm{NMR}\left(\mathrm{DMSO}-d_{6}\right): \delta 11.2,15.9,50.1,52.4,53.2,55.6$. Anal. calcd for $\mathrm{C}_{6} \mathrm{H}_{15} \mathrm{NO}$ (117.19): C, 61.49; H, 12.90; N, 11.95. Found C, 61.42; H, 12.85; N, 11.78.

\section{General procedure for the preparation of (2-chloroethyl)dialkylamine hydrochlorides (2)}

To a stirred solution of 2-(dialkylamino)ethanol $(0.1 \mathrm{~mol})$ in chloroform $(100 \mathrm{ml})$ a solution of thionyl chloride $(0.14 \mathrm{~mol})$ in chloroform $(10 \mathrm{ml})$ was added dropwise at $-5^{\circ} \mathrm{C}$. After removing an ice bath, the reaction mixture was allowed to reach the room temperature and then refluxed for $150 \mathrm{~min}$. After cooling, methanol $(20 \mathrm{ml})$ was added dropwise and the solution was stirred for 20 minutes. Then the solvents were evaporated in vacuo and the product was treated with 20 $\mathrm{ml}$ of methanol and evaporated to dryness again. The crude product was recrystallized and dried in vacuo at $90^{\circ} \mathrm{C}$.

(2-Chloroethyl)methylpropylamine hydrochloride (2b). Recrystallized from acetone and methanol (60:1). Yield $41 \%$, mp 167-169 ${ }^{\circ} \mathrm{C}$ (dec.); MS (ESI, $\left.m / z\right): 136.7[\mathrm{M}+\mathrm{H}]^{+}$; IR [ $\left.\mathrm{cm}^{-1}\right]$ : 2967, 2939, 2711, 2674, 2660, 2650, 1469, 1461, 1455, 1434, 1425, 759. ${ }^{1} \mathrm{H}-\mathrm{NMR}$ (DMSO- $d_{6}$ ): $\delta 0.88\left(\mathrm{t}, 3 \mathrm{H}, \mathrm{CH}_{3}, J=7.3 \mathrm{~Hz}\right) ; 1.71$ (sextet, $\left.2 \mathrm{H}, \mathrm{CH}_{2}, J=7.3 \mathrm{~Hz}\right) ; 2.75\left(\mathrm{~s}, 3 \mathrm{H}, \mathrm{CH}_{3}\right) ; 3.01-3.12$ $\left(\mathrm{m}, 2 \mathrm{H}, \mathrm{CH}_{2}\right) ; 3.38-3.47\left(\mathrm{~m}, 2 \mathrm{H}, \mathrm{CH}_{2}\right) ; 4.05\left(\mathrm{t}, 2 \mathrm{H}, \mathrm{CH}_{2}, J=7.3 \mathrm{~Hz}\right) ; 11.29(\mathrm{bs}, 1 \mathrm{H}, \mathrm{NH}) ;{ }^{13} \mathrm{C}-$ 
NMR (DMSO- $\left.d_{6}\right): \delta 10.7,16.5,37.2,39.1,55.1,56.5$. Anal. calcd for $\mathrm{C}_{6} \mathrm{H}_{15} \mathrm{Cl}_{2} \mathrm{~N}$ (172.1): C, 41.87; H, 8.79; N, 8.14. Found C, 42.03; H, 8.74; N, 8.22.

(2-Chloroethyl)ethylpropylamine hydrochloride (2d). Recrystallized from acetone and diethyl ether 10:7. Yield $66 \%$, mp 158-160 ${ }^{\circ} \mathrm{C}$ (dec.); MS (ESI, m/z): $150.7[\mathrm{M}+\mathrm{H}]^{+}$; IR [ $\left.\mathrm{cm}^{-1}\right]$ : 2969, 2939, 2664, 2631, 2600, 2502, 1469, 1460, 1398, 750. ${ }^{1} \mathrm{H}-\mathrm{NMR}\left(\mathrm{DMSO}-d_{6}\right): \delta \quad 0.89(\mathrm{t}$, $3 \mathrm{H}, \mathrm{CH}_{3}, J=7.5 \mathrm{~Hz}$ ); 1.23 (t, $3 \mathrm{H}, \mathrm{CH}_{3}, J=7.3 \mathrm{~Hz}$ ); 1.69 (sextet, $2 \mathrm{H}, \mathrm{CH}_{2}, J=7.5 \mathrm{~Hz}$ ); 2.98 $3.06\left(\mathrm{~m}, 2 \mathrm{H}, \mathrm{CH}_{2}\right) ; 3.10-3.21\left(\mathrm{~m}, 2 \mathrm{H}, \mathrm{CH}_{2}\right) ; 3.37-3.44\left(\mathrm{~m}, 2 \mathrm{H}, \mathrm{CH}_{2}\right) ; 4.06\left(\mathrm{t}, 2 \mathrm{H}, \mathrm{CH}_{2}, J=7.5\right.$ $\mathrm{Hz}$ ); 11.33 (bs, $1 \mathrm{H}, \mathrm{NH}) ;{ }^{13} \mathrm{C}-\mathrm{NMR}\left(\mathrm{DMSO}-d_{6}\right): \delta$ 8.2, 10.8, 16.2, 37.2, 50.0, 51.9, 52.7. Anal. calcd for $\mathrm{C}_{7} \mathrm{H}_{17} \mathrm{Cl}_{2} \mathrm{~N}$ (186.1): C, 45.17; H, 9.21; N, 7.53. Found C, 45.02; H, 9.31; N, 7.44.

General procedure for the preparation of [(2-dialkylamino)ethyl]isothiouronium chloride hydrochlorides (3)

A solution of (2-chloroethyl)dialkylamine hydrochloride $(70.9 \mathrm{mmol})$ and thiourea $(5.4 \mathrm{~g}, 70.9$ mmol) in dry ethanol $(50 \mathrm{ml})$ was refluxed for 7 hours. After cooling to room temperature, diethyl ether $(70 \mathrm{ml})$ was added and the solution was kept at $2{ }^{\circ} \mathrm{C}$ for 5 hours. The precipitated solid was separated by suction, washed with diethyl ether and dried in vacuo at $50^{\circ} \mathrm{C}$.

[2-(Ethylmethylamino)ethyl]isothiouronium chloride hydrochloride (3a). Recrystallized from dry ethanol and diethyl ether (10:6). Yield $86 \%$, mp 127-130 ${ }^{\circ} \mathrm{C}$ (dec.); MS (ESI, $\left.m / z\right)$ : $162.0[\mathrm{M}+\mathrm{H}]^{+}$; IR $\left[\mathrm{cm}^{-1}\right]: 2936,2690,2593,1649,1470,1444,1432,657 .{ }^{1} \mathrm{H}-\mathrm{NMR}$ (DMSO- $\left.d_{6}\right)$ : $\delta 1.23\left(\mathrm{t}, 3 \mathrm{H}, \mathrm{CH}_{3}, J=7.3 \mathrm{~Hz}\right) ; 2.75\left(\mathrm{~s}, 3 \mathrm{H}, \mathrm{CH}_{3}\right) ; 3.05-3.24\left(\mathrm{~m}, 2 \mathrm{H}, \mathrm{CH}_{2}\right) ; 3.29-3.37(\mathrm{~m}, 2 \mathrm{H}$, $\left.\mathrm{CH}_{2}\right) ; 3.68-3.70\left(\mathrm{~m}, 2 \mathrm{H}, \mathrm{CH}_{2}\right) ; 9.56\left(\mathrm{bs}, 4 \mathrm{H}, 2 \mathrm{NH}_{2}\right) ; 11.04$ (bs, $\left.1 \mathrm{H}, \mathrm{NH}\right) ;{ }^{13} \mathrm{C}-\mathrm{NMR}\left(\mathrm{DMSO}-d_{6}\right)$ : $\delta$ 8.75, 23.1, 38.3, 50.0, 52.6, 168.9 Anal. calcd for $\mathrm{C}_{6} \mathrm{H}_{17} \mathrm{Cl}_{2} \mathrm{~N}_{3} \mathrm{~S}(234.2)$ : C, 30.77; H, 7.32; N, 17.94; S, 13.69. Found C, 31.11; H, 7.30; N, 17.61; S, 13.96.

[2-(Methylpropylamino)ethyl]isothiouronium chloride hydrochloride (3b). Recrystallized from dry ethanol and diethyl ether (10:4). Yield $90 \%, \mathrm{mp} 108-110{ }^{\circ} \mathrm{C}$ (dec.); MS (ESI, $\mathrm{m} / z$ ): $176.0[\mathrm{M}+\mathrm{H}]^{+}$; IR $\left[\mathrm{cm}^{-1}\right]: 2174,1651,1413,668 .{ }^{1} \mathrm{H}-\mathrm{NMR}\left(\mathrm{DMSO}-d_{6}\right): \delta 0.89\left(\mathrm{t}, 3 \mathrm{H}, \mathrm{CH}_{3}, J=\right.$ $7.4 \mathrm{~Hz}$ ); 1.69 (sextet, 2H, $\mathrm{CH}_{2}, J=7.4 \mathrm{~Hz}$ ); 2.77 (s, 3H, $\left.\mathrm{CH}_{3}\right) ; 3.20$ (t, 2H, $\mathrm{CH}_{2}, J=7.4 \mathrm{~Hz}$ ); 3.33 (t, 2H, $\mathrm{CH}_{2}, J=7.0 \mathrm{~Hz}$ ); 3.71 (t, 2H, $\mathrm{CH}_{2}, J=7.0 \mathrm{~Hz}$ ); 9.51 (bs, 4H, 2NH ); 10.95 (bs, $1 \mathrm{H}$, $\mathrm{NH}) ;{ }^{13} \mathrm{C}-\mathrm{NMR}\left(\mathrm{DMSO}-d_{6}\right): \delta 10.8,16.7,24.0,38.9,53.2,56.3,168.9$. Anal. calcd for $\mathrm{C}_{7} \mathrm{H}_{19} \mathrm{Cl}_{2} \mathrm{~N}_{3} \mathrm{~S}$ (248.2): C, 33.87; H, 7.72; N, 16.93; S, 12.92. Found C, 33.96; H, 7.96; N, 17.10; $\mathrm{S}, 13.14$.

[2-(Isopropylmethylamino)ethyl]isothiouronium chloride hydrochloride (3c). Recrystallized from dry ethanol. Yield $84 \%$, mp 202-203 ${ }^{\circ} \mathrm{C}$ (dec.); MS (ESI, $\left.m / z\right): 176.0[\mathrm{M}+\mathrm{H}]^{+}$; IR $\left[\mathrm{cm}^{-1}\right]$ : 2974, 2743, 2708, 2696, 1652, 1638, 1441, 1423, 1412, 1392, 1144. ${ }^{1}$ H-NMR (DMSO- $d_{6}$ ): $\delta$ $1.23\left(\mathrm{~m}, 6 \mathrm{H}, 2 \mathrm{CH}_{3}\right) ; 2.67\left(\mathrm{~s}, 3 \mathrm{H}, \mathrm{CH}_{3}\right) ; 2.67-3.37\left(\mathrm{~m}, 2 \mathrm{H}, \mathrm{CH}_{2}\right) ; 3.60$ (hept, $1 \mathrm{H}, \mathrm{CH}, J=6.6$

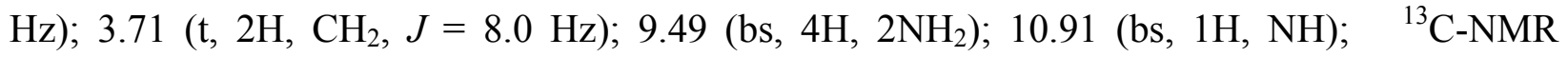
$\left(\mathrm{DMSO}-d_{6}\right): \delta 18.5,24.4,34.0,50.8,55.92,56.1,169.0$. Anal. calcd for $\mathrm{C}_{7} \mathrm{H}_{19} \mathrm{Cl}_{2} \mathrm{~N}_{3} \mathrm{~S}(248.2)$ : C, 33.87; H, 7.72; N, 16.93; S, 12.92. Found C, 34.02; H, 7.58; N, 17.27; S, 13.11. 
[2-(Ethylpropylamino)ethyl]isothiouronium chloride hydrochloride (3d). Recrystallized from dry ethanol and diethyl ether (2:1). Yield $74 \%$, mp $170{ }^{\circ} \mathrm{C}$ (dec.); MS (ESI, $\mathrm{m} / \mathrm{z}$ ): 190.0 $[\mathrm{M}+\mathrm{H}]^{+}$; IR $\left[\mathrm{cm}^{-1}\right]: 2966,2723,2620,2573,1653,1458,1452,1429,676 .{ }^{1} \mathrm{H}-\mathrm{NMR}$ (DMSO- $\left.d_{6}\right)$ : $\delta \quad 0.90\left(\mathrm{t}, 3 \mathrm{H}, \mathrm{CH}_{3}, J=7.4 \mathrm{~Hz}\right) ; 1.23\left(\mathrm{t}, 3 \mathrm{H}, \mathrm{CH}_{3}, J=7.2 \mathrm{~Hz}\right.$ ); 1.68 (sextet, $2 \mathrm{H}, \mathrm{CH}_{2}, J=7.4$ $\mathrm{Hz}) ; 3.05$ (t, $\left.2 \mathrm{H}, \mathrm{CH}_{2}, J=8.0 \mathrm{~Hz}\right) ; 3.14-3.18\left(\mathrm{~m}, 2 \mathrm{H}, \mathrm{CH}_{2}\right) ; 3.31$ (t, 2H, $\left.\mathrm{CH}_{2}, J=7.3 \mathrm{~Hz}\right)$; 3.71 -

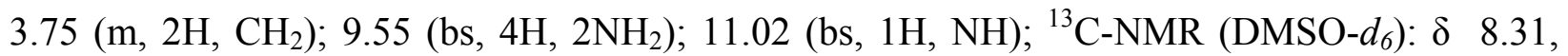
10,8, 16.4, 23.9, 46.7, 50.0, 52.5, 168.9 . Anal. calcd for $\mathrm{C}_{8} \mathrm{H}_{21} \mathrm{Cl}_{2} \mathrm{~N}_{3} \mathrm{~S}$ (262.2): C, 36.64; $\mathrm{H}, 8.07$; N, 16.02; S, 12.23. Found C, 36.78; H, 8.01; N, 16.04; S, 12.22.

[2-(Ethylisopropylamino)ethyl]isothiouronium chloride hydrochloride (3e). Recrystallized from dry ethanol and diethyl ether (10:8). Yield $86 \%$, mp 171-173 ${ }^{\circ} \mathrm{C}$ (dec.); MS (ESI, $\left.m / z\right)$ : $190.0[\mathrm{M}+\mathrm{H}]^{+}$; IR $\left[\mathrm{cm}^{-1}\right]: 2961,2729,2658,2641,1654,1448,1430 .{ }^{1} \mathrm{H}-\mathrm{NMR}$ (DMSO-d $): \delta$ 1.26-1.31 (m, 9H, 3CH $)$; 3.17 (q, 2H, $\left.\mathrm{CH}_{2}, J=6.8 \mathrm{~Hz}\right)$; 3.66-3.75 (m, 2H, 2CH); 3.66 (hept, 1H, $\mathrm{CH}, J=6.6 \mathrm{~Hz}) ; 3.78-3.83\left(\mathrm{~m}, 2 \mathrm{H}, \mathrm{CH}_{2}\right) ; 9.54\left(\mathrm{bs}, 4 \mathrm{H}, 2 \mathrm{NH}_{2}\right) ; 10.82(\mathrm{bs}, 1 \mathrm{H}, \mathrm{NH}) ;{ }^{13} \mathrm{C}-\mathrm{NMR}$ $\left(\mathrm{DMSO}-d_{6}\right): \delta 9.8,15.8,16.1,24.7,44.2,47.8,53.6,169.1$. Anal. calcd for $\mathrm{C}_{8} \mathrm{H}_{21} \mathrm{Cl}_{2} \mathrm{~N}_{3} \mathrm{~S}$ (262.2): C, 36.64; H, 8.07; N, 16.02; S, 12.23. Found C, 37.02; H, 8.03; N, 16.02; S, 12.33 .

[2-(Dipropylamino)ethyl]isothiouronium chloride hydrochloride (3f). Recrystallized from dry ethanol and diethyl ether (2:1). Yield $72 \%$, mp $177^{\circ} \mathrm{C}$ (dec.); MS (ESI, $\left.m / z\right): 204.0[\mathrm{M}+\mathrm{H}]^{+}$; IR $\left[\mathrm{cm}^{-1}\right.$ ]: $2975,2962,2937,2597,2560,1656,1459,1428,723 .{ }^{1} \mathrm{H}-\mathrm{NMR}$ (DMSO- $\left.d_{6}\right): \delta 0.90$ (t, $6 \mathrm{H}, 2 \mathrm{CH}_{3}, J=7.5 \mathrm{~Hz}$ ); 1.69 (sextet, $4 \mathrm{H}, 2 \mathrm{CH}_{2}, J=7.5 \mathrm{~Hz}$ ); 3.06 (t, 4H,2 $\mathrm{CH}_{2}, J=8.0 \mathrm{~Hz}$ ); $3.31\left(\mathrm{t}, 2 \mathrm{H}, \mathrm{CH}_{2}, J=7.3 \mathrm{~Hz}\right.$ ); 3.75 (t, 2H, $\left.\mathrm{CH}_{2}, J=7.5 \mathrm{~Hz}\right) ; 9.52$ (bs, 4H, 2NH ); 11.02 (bs, $1 \mathrm{H}$, $\mathrm{NH}) ;{ }^{13} \mathrm{C}-\mathrm{NMR}$ (DMSO- $\left.d_{6}\right): \delta 10.8,16.4,23.9,50.6,53.2,169.0$. Anal. calcd for $\mathrm{C}_{9} \mathrm{H}_{23} \mathrm{Cl}_{2} \mathrm{~N}_{3} \mathrm{~S}$ (276.3): C, 39.13; H, 8.39; N, 15.21; S, 11.61. Found C, 39.42; H, 8.29; N, 15.16; S, 11.73.

\section{[2-(Isopropylpropylamino)ethyl]isothiouronium chloride hydrochloride (3g).}

Recrystallized from dry ethanol and diethyl ether (2:1). Yield $78 \%$, mp 164-166 ${ }^{\circ} \mathrm{C}$ (dec.); MS (ESI, $m / z): 204.0[\mathrm{M}+\mathrm{H}]^{+}$; IR $\left[\mathrm{cm}^{-1}\right.$ ]: 2984, 2972, 1655, 1440, 1429, 1410. ${ }^{1} \mathrm{H}-\mathrm{NMR}$ (DMSO- $d_{6}$ ): $\delta 0.91\left(\mathrm{t}, 3 \mathrm{H}, \mathrm{CH}_{3}, J=7.3 \mathrm{~Hz}\right) ; \delta 1.23\left(\mathrm{~d}, 6 \mathrm{H}, 2 \mathrm{CH}_{3}, J=6.4 \mathrm{~Hz}\right) ; 1.74\left(\right.$ sextet, $2 \mathrm{H}, \mathrm{CH}_{2}, J=7.3$ $\mathrm{Hz})$; 2.97-3.08 (m, 2H, CH$)$; 3.29-3.31 (m, 2H, $\left.\mathrm{CH}_{2}\right)$; $3.65(\mathrm{~h}, 1 \mathrm{H}, \mathrm{CH}, J=6.6 \mathrm{~Hz}) ; 3.78$ (t, 2H, $\mathrm{CH}_{2}, J=7.3 \mathrm{~Hz}$ ); 9.54 (bs, 4H, 2NH ); 10.74 (bs, $\left.1 \mathrm{H}, \mathrm{NH}\right) ;{ }^{13} \mathrm{C}-\mathrm{NMR}$ (DMSO- $d_{6}$ ): $\delta 10.9,15.5$, 16.3, 17.9, 24.6, 48.7, 50.4, 54.2, 169.2. Anal. calcd for $\mathrm{C}_{9} \mathrm{H}_{23} \mathrm{Cl}_{2} \mathrm{~N}_{3} \mathrm{~S}$ (276.3): C, 39.13; $\mathrm{H}, 8.39$; N, 15.21; S, 11.61. Found C, 39.24; H, 8.56; N, 15.24; S, 11.76.

\section{General procedure for the preparation of 2-(dialkylamino)ethanethiols hydrochlorides (4)}

(All used solvents have to be degassed by argon. Separation of solid products by suction has to be carried out under nitrogen or argon, without the admittance of humid air. The products are highly hygroscopic!)

To a stirred solution of [(2-dialkylaminoethyl)]isothiouronium chloride hydrochloride $(0.05 \mathrm{~mol})$ in water $(19 \mathrm{ml})$ was added solution of $\mathrm{NaOH}(4.0 \mathrm{~g}, 0.1 \mathrm{~mol})$ in water $(12 \mathrm{ml})$. After 10 minutes of stirring the reaction mixture was saturated with $\mathrm{NaCl}$ and thiol was extracted with diethyl ether $(3 \times 10 \mathrm{ml})$. The ethereal solution was dried with $\mathrm{MgSO}_{4}$, filtered off and diethyl ether was evaporated in vacuo $\left(250\right.$ torr $\left./ 40^{\circ} \mathrm{C}\right)$. Oily product was then purified by distillation in vacuo (at 
30 torr). Obtained product was dissolved in $20 \mathrm{ml}$ of diethyl ether and ethanolic hydrogen chloride was added dropwise with stirring and cooling in an ice bath. The precipitated 2(dialkylamino)ethanethiol hydrochloride was separated by suction and dried in vacuo at $50^{\circ} \mathrm{C}$.

2-(Ethylmethylamino)ethanethiol hydrochloride (4a). Recrystallized from diethyl ether and dry ethanol (1:2). Yield $20 \%$, mp 127-129 ${ }^{\circ} \mathrm{C}$ (dec.); MS (ESI, $\left.m / z\right): 120.1[\mathrm{M}+\mathrm{H}]^{+}$; IR [ $\left.\mathrm{cm}^{-1}\right]$ : 2941, 2684, 2662, 2477, 1473, 1434, 1426, 1396, 1022. ${ }^{1} \mathrm{H}-\mathrm{NMR}$ (DMSO- $\left.d_{6}\right)$ : ${ }^{1} \mathrm{H}-\mathrm{NMR}$ (DMSO- $\left.d_{6}\right): \delta 1.22\left(\mathrm{~s}, 3 \mathrm{H}, \mathrm{CH}_{3}, J=7.4 \mathrm{~Hz}\right) ; 2.68\left(\mathrm{~s}, 3 \mathrm{H}, \mathrm{CH}_{3}\right) ; 2.85\left(\mathrm{t}, 2 \mathrm{H}, \mathrm{CH}_{2}, J=7.1 \mathrm{~Hz}\right)$; 3.03-3.25 (m, 5H, 2 $\mathrm{CH}_{2}+\mathrm{SH}$ ); 11.04 (bs, $1 \mathrm{H}, \mathrm{NH}$ ); ${ }^{13} \mathrm{C}-\mathrm{NMR}$ (DMSO-d $): \delta \quad 8.66,17.7,38.3$, 49.8, 56.6. Anal. calcd for $\mathrm{C}_{5} \mathrm{H}_{14} \mathrm{ClNS}$ (155.7): C, 38.57; H, 9.06; N, 9.00; S, 20.60. Found C, 38.44; H, 9.09; N, 8.93; S, 20.67.

2-(Methylpropylamino)ethanethiol hydrochloride (4b). Recrystallized from diethyl ether and dry ethanol (1:2). Yield $24 \%$, mp 153-155 ${ }^{\circ} \mathrm{C}$ (dec.); MS (ESI, $\left.m / z\right): 134.1[\mathrm{M}+\mathrm{H}]^{+}$; IR [ $\left.\mathrm{cm}^{-1}\right]$ : 2963, 2939, 2680, 1476, 1458, 1427, 957. ${ }^{1} \mathrm{H}-\mathrm{NMR}$ (DMSO-d $)$ : $\delta 0.89$ (t, 3H, $\mathrm{CH}_{3}, J=7.4 \mathrm{~Hz}$ ); 1.70 (sextet, $2 \mathrm{H}, \mathrm{CH}_{2}, J=7.4 \mathrm{~Hz}$ ); $2.72\left(\mathrm{~s}, 3 \mathrm{H}, \mathrm{CH}_{3}\right) ; 2.9-3.37\left(\mathrm{~m}, 7 \mathrm{H}, 3 \mathrm{CH}_{2}+\mathrm{SH}\right) ; 11.06$ (bs, $1 \mathrm{H}, \mathrm{NH}) ;{ }^{13} \mathrm{C}-\mathrm{NMR}\left(\mathrm{DMSO}-d_{6}\right): \delta$ 16.6, 17.6, 23.7, 54.0, 56.2, 57.2. Anal. calcd for $\mathrm{C}_{6} \mathrm{H}_{16} \mathrm{ClNS}$ (169.7): C, 42.46; H, 9.50; N, 8.25; S, 18.89. Found C, 42.41; H, 9.47; N, 8.31; S, 18.74 .

2-(Isopropylmethylamino)ethanethiol hydrochloride (4c). Recrystallized from diethyl ether and dry ethanol (1:2). Yield $21 \%$, mp $51{ }^{\circ} \mathrm{C}$ (dec.); MS (ESI, $\left.m / z\right): 134.1[\mathrm{M}+\mathrm{H}]^{+}$; IR $\left[\mathrm{cm}^{-1}\right]$ : 2977, 2951, 2690, 2471, 1627, 1468, 1086. ${ }^{1} \mathrm{H}-\mathrm{NMR}$ (DMSO- $\left.d_{6}\right): \delta 1.23\left(\mathrm{~d}, 6 \mathrm{H}, 2 \mathrm{CH}_{3}, J=4.4\right.$ $\mathrm{Hz}) ; 2.61\left(\mathrm{~s}, 3 \mathrm{H}, \mathrm{CH}_{3}\right) ; 2.85-2.95\left(\mathrm{~m}, 2 \mathrm{H}, \mathrm{CH}_{2}\right) ; 3.10-3.20\left(\mathrm{~m}, 3 \mathrm{H}, \mathrm{CH}_{2}+\mathrm{SH}\right) ; 3.49$ (h, $1 \mathrm{H}, \mathrm{CH}, J$ $=6.8 \mathrm{~Hz}) ; 10.91(\mathrm{bs}, 1 \mathrm{H}, \mathrm{NH}) ;{ }^{13} \mathrm{C}-\mathrm{NMR}\left(\mathrm{DMSO}-d_{6}\right): \delta \quad 18.1,34.3,34.7,54.4,55,9$. Anal. calcd for $\mathrm{C}_{6} \mathrm{H}_{16} \mathrm{CINS}$ (169.7): C, 42.46; H, 9.50; N, 8.25; S, 18.89. Found C, 42.26; H, 9.69; N, $8.23 ; \mathrm{S}, 18.74$.

2-(Ethylpropylamino)ethanethiol hydrochloride (4d). Recrystallized from diethyl ether and dry ethanol (1:1). Yield $26 \%$, mp $111^{\circ} \mathrm{C}$ (dec.); MS (ESI, $\left.m / z\right): 148.1[\mathrm{M}+\mathrm{H}]^{+}$; IR [ $\left.\mathrm{cm}^{-1}\right]: 2964$, 2934, 2702, 2668, 2636, 1463, 1438, 1394, 1038, 1026, 978. ${ }^{1} \mathrm{H}-\mathrm{NMR}$ (DMSO- $d_{6}$ ): $\delta 0.89$ (t, 3H, $\mathrm{CH}_{3}, J=7.2 \mathrm{~Hz}$ ); 1.22 (t, 3H, $\mathrm{CH}_{3}, J=7.3 \mathrm{~Hz}$ ); 1.68 (sextet, $2 \mathrm{H}, \mathrm{CH}_{2}, J=7.3 \mathrm{~Hz}$ ); 2.85 (t, 2H, $\mathrm{CH}_{2}, J=8.0 \mathrm{~Hz}$ ); 3.96 (q, 2H, $\mathrm{CH}_{2}, J=7.0 \mathrm{~Hz}$ ); 3.06-3.2 (m, 5H, 2CH $\left.\mathrm{CH}_{2} \mathrm{SH}\right) ; 10.99$ (bs, $1 \mathrm{H}$, $\mathrm{NH}) ;{ }^{13} \mathrm{C}-\mathrm{NMR}\left(\mathrm{DMSO}-d_{6}\right): \delta 8.2,10.8,16.3,17.5,46.7,52.6,53.7$. Anal. calcd for $\mathrm{C}_{7} \mathrm{H}_{18} \mathrm{ClNS}$ (183.7): C, 45.76; H, 9.87; N, 7.62; S, 17.45. Found C, 45.82; H, 9.78; N, 7.55; S, 17.35.

2-(Ethylisopropylamino)ethanethiol hydrochloride (4e). Recrystallized from ethanol and diethyl ether (1:2). Yield $42 \%$, mp $117-119{ }^{\circ} \mathrm{C}$ (dec.); MS (ESI, $\left.m / z\right): 148.1[\mathrm{M}+\mathrm{H}]^{+}$; IR [ $\left.\mathrm{cm}^{-1}\right]$ : 2943, 2691, 2640, 2430, 1474, 1462, 1443, 1434, 1404, 1383, 1135. ${ }^{1}$ H-NMR (DMSO-d 6 ): $\delta$ 1.26-1.31 (m, 9H, $\left.3 \mathrm{CH}_{3}\right) ; 2.83\left(\mathrm{t}, 2 \mathrm{H}, \mathrm{CH}_{2}, J=7.3 \mathrm{~Hz}\right) ; 3.1-3.31\left(\mathrm{~m}, 5 \mathrm{H}, 2 \mathrm{CH}_{2}+\mathrm{SH}\right) ; 3.57$ (h, $1 \mathrm{H}, \mathrm{CH}, J=6.6 \mathrm{~Hz}) ; 10.70$ (bs, $1 \mathrm{H}, \mathrm{NH}) ;{ }^{13} \mathrm{C}-\mathrm{NMR}\left(\mathrm{DMSO}-d_{6}\right): \delta 9.5,15.7,16.3,18.7,44.6$, 51.4, 53.7. Anal. calcd for $\mathrm{C}_{7} \mathrm{H}_{18} \mathrm{ClNS}$ (183.7): C, 45.76; H, 9.87; N, 7.62; S, 17.45. Found C, 45.97; H, 9.67; N, 7.46; S, 17.28. 
2-(Dipropylamino)ethanethiol hydrochloride (4f). Recrystallized from diethyl ether and dry ethanol (1:1). Yield $19 \%$, mp 98-100 ${ }^{\circ} \mathrm{C}$ (dec.); MS (ESI, $\left.m / z\right): 162.1[\mathrm{M}+\mathrm{H}]^{+}$; IR [ $\left.\mathrm{cm}^{-1}\right]: 2965$, 2938, 2703, 2628, 2528, 2465, 1474, 1046, 980, 752. ${ }^{1} \mathrm{H}-\mathrm{NMR}\left(\mathrm{DMSO}-d_{6}\right): \delta 0.89$ (t, $6 \mathrm{H}, 2 \mathrm{CH}_{3}$, $J=7.3 \mathrm{~Hz}$ ); $\delta 1.68$ (sextet, $4 \mathrm{H}, 2 \mathrm{CH}_{2}, J=7.3 \mathrm{~Hz}$ ); 2.86 (bs, $\left.2 \mathrm{H}\right) ; 2.97$ (t, 4H, 2CH $2, J=7.3 \mathrm{~Hz}$ ); 3.10-3.22 (m, 3H, SH+CH$) ; 10.90(\mathrm{bs}, 1 \mathrm{H}, \mathrm{NH}) ;{ }^{13} \mathrm{C}-\mathrm{NMR}\left(\mathrm{DMSO}-d_{6}\right): \delta 10.8,16.3,17.5$, 51.0, 53.3, 54.3. Anal. calcd for $\mathrm{C}_{8} \mathrm{H}_{20} \mathrm{ClNS}$ (197.8): C, 48.58; H, 10.19; N, 7.08; S, 16.21 . Found C, 48.54; H, 10.29; N, 7.13; S, 16.27.

2-(Isopropylpropylamino)ethanethiol hydrochloride (4g). Recrystallized from diethyl ether and dry ethanol (1:1). Yield $56 \%$, mp 140-141 ${ }^{\circ} \mathrm{C}$ (dec.); MS (ESI, $\left.m / z\right): 162.1[\mathrm{M}+\mathrm{H}]^{+}$; IR [ $\mathrm{cm}^{-}$ ${ }^{1}$ ]: 2980, 2964, 2939, 2675, 2634, 2526, 2431 1469, 1453, 1448, 1379, 1136, 980. ${ }^{1} \mathrm{H}-\mathrm{NMR}$ (DMSO- $\left.d_{6}\right): \delta 0.90\left(\mathrm{t}, 3 \mathrm{H}, \mathrm{CH}_{3}, J=7.4 \mathrm{~Hz}\right) ; \delta 1.26\left(\mathrm{~d}, 6 \mathrm{H}, 2 \mathrm{CH}_{3}, J=6.3 \mathrm{~Hz}\right.$ ); 1.75 (sextet, 2H, $\left.\mathrm{CH}_{2}, J=8.4 \mathrm{~Hz}\right) ; 2.90-3.11\left(\mathrm{~m}, 4 \mathrm{H}, 2 \mathrm{CH}_{2}\right) ; 3.10-3.28\left(\mathrm{~m}, 3 \mathrm{H}, \mathrm{SH}+\mathrm{CH}_{2}\right) ; 3.56(\mathrm{~h}, 1 \mathrm{H}, \mathrm{CH}, J=$ $6.6 \mathrm{~Hz}) ; 10.70(\mathrm{bs}, 1 \mathrm{H}, \mathrm{NH}) ;{ }^{13} \mathrm{C}-\mathrm{NMR}\left(\mathrm{DMSO}-d_{6}\right): \delta 11.0,15.9,16.0,17.5,18.7,51.0,52.2$, 54.1. Anal. calcd for $\mathrm{C}_{8} \mathrm{H}_{20} \mathrm{ClNS}$ (197.8): C, 48.58; H, 10.19; N, 7.08; S, 16.21. Found C, 48.61; H, 10.26; N, 7.05; S, 16.38 .

\section{Acknowledgements}

The authors gratefully acknowledge financial support by The Organization for the Prohibition of Chemical Weapons, The Hague, The Netherlands (No. L/ICA/ICB/84366/04), The State Office for Nuclear Safety, Czech Republic, and the Ministry of Education, Youth and Sports, Czech Republic (MSM6198959216).

\section{References}

1. Papoušková, B.; Bednář, P.; Barták, P.; Fryčák, P.; Ševčík, J.; Stránský, Z.; Lemr, K.; J. Sep. Sci. 2006; 29, 1531.

2. The Convention on the Prohibition of the Development, Production, Stockpiling and Use of Chemical Weapons and on their Destruction, Organization for the Prohibition of Chemical Weapons (OPCW), The Hague 1994 (www.opcw.org).

3. Yang, Y. Acc. Chem. Res. 1999, 32, 109.

4. Copper, C. L.; Collins, G. E. Electrophoresis 2004, 25, 897.

5. Joshi, K. A.; Prouza, M.; Kum, M.; Wang, J.; Tang, J.; Haddon, R.; Chen, W.; Mulchandani, A. Anal. Chem. 2006, 78, 331.

6. Gilman, H.; Plunkett, M. A.; Tolman, L.; Fullhart, L.; Broadbent, H. S. J. Am. Chem. Soc. 1945, 67, 1845.

7. Weijlard, J.; Tishler, M. US 2,642,428 (1953).

8. Albertson, N. F; Clinton, R. O. J. Am. Chem. Soc. 1945, 67, 1222. 
9. Clinton, R. O.; Salvador, U. J.; Laskowski, S. C.; Suter, C. M. J. Am. Chem. Soc. 1948, 70, 950.

10. Snyder, H. R.; Stewart, J. M.; Ziegler, J. B. J. Am. Chem. Soc. 1947, 69, 2672.

11. Hansen, B. Acta Chem. Scand. 1953, 13, 151.

12. Wineman, R. J.; Gollis, M. H.; James, J. C.; Pomponi, A. M. J. Org. Chem. 1962, 27, 4222.

13. Reynolds, D. D.; Fields, D. L.; Johnson, D. L. J. Org. Chem. 1961, 26, 5125.

14. Straley, J. M.; Wallace, D. J.; Fisher, J. G. US 3,236,843 (1966).

15. Fields, D. L.; Reynolds, D. D. US 3,335,161 (1967).

16. Reynolds, D. D. US 3,232,936 (1966).

17. Parkkari, J. K.; Bannard, R. A. B.; Coleman, I. W. Can. J. Chem. 1965, 43, 3119.

18. Wright, J. B.; Lincoln, E. H.; Heinzelmann, R. V.; Hunter, J. H. J. Am. Chem. Soc. 1950, 72, 3536.

19. Soulal, M. J.; Twamley, E. A. GB 1,441,749 (1976).

20. Davis, F. A.; Ray, J. K.; Kasperowicz, S.; Przeslawski, R. M.; Durst, H. D. J. Org. Chem. 1992, 57, 2594.

21. Mahmoudkani, A. M.; Langer, V. Acta Crystallogr. Sect. C: Cryst. Struct. Commun. 1999, 55, 1163.

22. Doyle, F. P.; Mehta, M. D.; Ward, R.; Bainbridge, J.; Brown, D. M. J. Med. Chem. 1965, 8, 571. 